\title{
Paper Alert
}

\section{Checkpoint Inhibitors for Advanced Bladder Cancer}

\author{
Edward M. Messing* \\ University of Rochester Medical Center, Rochester, NY, USA
}

Metastatic urothelial cancer (UC) recurring after cisplatin based combination chemotherapy represents an incurable condition for which treatment with second line chemotherapy is almost always unsuccessful in achieving prolonged remissions. The recent introduction into the therapeutic armamentarium of immune checkpoint inhibitors can potentially be a "game changer", although success currently remains limited.

$\mathrm{UC}$ is one of the cancers with many somatic mutations, along with melanoma and non-small cell lung cancer (NSCLC) [1] and there are data associating mutational burden with response to checkpoint inhibitors in NSCLC [2] and melanoma [3]. Initially for UC this approach focused on cytotoxic T lymphocyte-associated protein 4 (CTLA4) inhibitors, which blocked the immune system's recognition of tumor-induced molecular signals which diminish immune responses to cancers. The CTLA-4 inhibitor, ipilimumab, caused more immune $\mathrm{T}$ lymphocytes to infiltrate UCs, but did not result, alone or combined with Gemcitabine- cisplatin therapy, in prolonging survival [4]. However, attacking another immune checkpoint, programmed death receptor and programmed death receptor ligand 1 (PD-1 and PD-L1, respectively) has effected prolonged survival in some UC patients. We will review recent studies using this approach, which holds great promise.

Powles, et al., [5] examined activity of the high affinity human anti-PD-L1 antibody, MPDL3280A (atezolizumab), in patients with metastatic UC in

\footnotetext{
${ }^{*}$ Correspondence to: Edward M. Messing, University of Rochester Medical Center, Rochester, NY, USA. Tel.: +1 585275 3345; E-mail: Edward.messing@urmc.rochester.edu.
}

a Phase 1 expansion study. PD-L1 is often expressed in activated $\mathrm{T}$ cells and atezolizumab's $\mathrm{Fc}$ domain has been modified to eliminate antibody dependent cellular cytotoxicity to prevent depletion of T cells expressing PD-L1. Patients with metastatic UC who had previously received cisplatin or carboplatin containing chemotherapy had tumor infiltrating lymphocytes (TILs) in primary or metastatic cancers studied for PD-LI expression by immunohistochemistry (IHC score 0-3) in both archived or fresh tissue. Most patients had failed more than one chemotherapy regimen and had several other adverse clinical features. The initial cohort had grade 2 or 3 PDLI expression on TILs, but eventually the study was expanded to include patients with no or weak staining. Patients received IV infusions of $15 \mathrm{mg} / \mathrm{kg}$ atezolizumab over 30-60 minutes every three weeks for 16 treatments or up to one year, stopping for intolerance or disease progression. Adverse events were common, but usually minor. The objective response rate (ORR) was $34 \%$ in 87 evaluable patients, but was $50 \%$ in 46 patients with grade 2-3 staining with nine complete responses (CRs). Several of the responses were ongoing at publication. This study led the Food and Drug Administration (FDA) to approve this drug with "breakthrough therapy" designation. Interleukin 18 (IL-18) and Interferon-alpha (IFN-alpha) (stimulated by IL-18) levels transiently increased in all patients as did CD8 + HLA-Dr+Ki67 + T cells. No immune markers correlated with efficacy.

These findings were confirmed in a large $(N=310)$ phase II study of patients with locally/regionally advanced (stage T4b and any $\mathrm{N}$ or any $\mathrm{T}$ and $\mathrm{N} 2,3$ ) or metastatic (M1) UC whose disease had progressed after cisplatin or carboplatin based chemotherapy. 
Patients received $1200 \mathrm{mg}$ atezolizumab IV every 3 weeks [6]. A 15\% ORR was achieved in all patients, with $26 \%$ in the grade 2 and 3 PD-L1 staining group, and $10 \%$ in the grade 1 staining group, with $11 \%$ CRs in the former and $2 \%$ in the latter. Median overall survival had not been reached at publication (median follow-up 11 months) in the grade 2, 3 staining group, and was 6.7 months in the grade 1 staining group. As with the Powles' et al. study [5], grade 34 adverse events occurred in $16 \%$ (most commonly fatigue), with immune mediated grade 3-4 adverse events (pneumonitis, rash, dyspnea and liver toxicity) occurring in $5 \%$.

Massard and co-workers [7], in a similar cohort of 61 patients reported results of a different antiPD-L1 monoclonal antibody, durvalumab, defining PD-L1 positivity as $>25 \%$ of tumor cells or TILs staining $(N=40)$ and negative for $<25 \%$ of cells staining, $(N=21) .10 \mathrm{mg} / \mathrm{kg}$ were infused every 2 weeks, with grade 3 toxicity in $<15 \%$ of patients (mostly fatigue) and no grade 4 or 5 toxicity. While median follow-up was only 4.3 months, the ORR was $46 \%$ in PD-L1 positive patients and 0 in PD-L1 negative patients. As with atezolizumab, responses were ongoing, including in one patient who discontinued treatment because of grade 3 renal toxicity.

While still only appearing as an American Society of Clinical Oncology's annual meeting abstract [8], Plimack and co-workers studied a PD-1 inhibitor, pembrolizumab, with similar patients with $>1+$ PDL1 staining on tumor cells or TILs, reported an ORR in $28 \%$ of 33 evaluable patients and CRs in $9 \%$.

Because of the brief follow-up in all of these studies, uncertainty of predictive markers and absence of phase III data, there's reason for some caution in interpreting these results, but there is also considerable reason for optimism [5-8].

Not surprisingly, acquired resistance to PD-1/PDL1 blockade also occurs. In analyzing four patients with melanoma who initially responded to pembrolizumab for months to years and then progressed, whole-exome sequencing of baseline and recurrent cancers showed that loss of function mutations of genes encoding for IFY receptor associated JAK1 or JAK2, resulted in loss of response to IF- $\mathrm{Y}$ including insensitivity to its antiproliferative effects on cancer cells. Another patient had a truncating mutation of the gene encoding beta-2-microglobulin (B2M) leading to loss of surface expression of major histocompatibility complex (MHC) class 1 antigen in a patient whose baseline tumor already lacked
MHC class II expression [9, 10]. Undoubtedly, similar and other escape mechanisms are certain to be seen in UC as well. Anticipating this event, studies are being designed or are already ongoing of additional PD-1 or PD-L1 inhibitors of combinations of PD-L1 or PD-1 inhibitors with chemotherapy or molecular targeted therapies (both in previously treated or untreated patients), or of two checkpoint inhibitors having different immune targets (e.g. anti CTLA-4 and anti PD-1, PD-L1). The last has shown increased efficacy but increased toxicity in melanoma patients [11].

Thus, although experience is limited, the development of immune checkpoint inhibitors has ushered in a new era of hope for patients with advanced UC. Undoubtedly, these approaches will soon be tested in patients with earlier stages of UC as well.

\section{REFERENCES}

[1] Alexandrov LB, Nik-Zainal S, Wedge DC, et al. Signatures of mutational processes in human cancer. Nature 2013;500:415-21.

[2] Rizvi NA, Hellmann MD, Snyder A, et al. Mutational landscape determines sensitivity to PD-1 blockade in non-small cell lung cancer. Science 2015;348:124-8.

[3] Snyder A, Makarov V, Merghoub T, et al. Genetic basis for clinical response to CTLA-4 blockade in melanoma. N Engl J Med 2014;371:2189-99.

[4] Carthon BC, Wolchok JD, Yuan J, et al. Preoperative CTLA-4 blockade: Tolerability and immune monitoring in the setting of a presurgical clinical trial. Clin Cancer Res 2010;16:2861-71.

[5] Powles T, Eder JP, Fine GD, et al. MPDL3280A (anti-PDL1) treatment leads to clinical activity in metastatic bladder cancer. Nature 2014;515:558-63.

[6] Rosenberg JE, Hoffman-Censitts J, Powles T, et al. Atezolizumab in patients with locally advanced and metastatic urothelial cancer who have progressed following treatment with platinum-based chemotherapy: A single-arm, multicenter, phase 2 trial. The Lancet 2016;387:1909-20.

[7] Massard C, Gordon MS, Sharma S, et al. Safety and efficacy of Durvalumab (MEDI4736), an anti-programmed cell death ligand-1 immune checkpoint inhibitor, in patients with advanced urothelial bladder cancer. J Clin Onc 2016; 34:3119-25.

[8] Plimack ER, Bellmunt J, Gupta S, et al. Pembrolizumab (MK-3475) for advanced urothelial cancer: Updated results and biomarker analysis from KEYNOTE-012. J Clin Oncol 2015;33(suppl): Abstract 4502.

[9] Zaretsky JM, Garcia-Diaz A, Shin D, et al. Mutations associated with acquired resistance to PD-1 blockade in melanoma. NEJM 2016;375:819-29.

[10] Bifulco CB, Urba WJ. Unmasking PD-1 resistance by nextgeneration sequencing. NEJM 2016;375:888-9.

[11] Zibelman M, Plimack ER. Checkpoint inhibitors and urothelial carcinoma: The translational paradigm. Oncology 2016;30:160-76. 